\title{
Acid-Promoted Synthesis of Imidazolyl-Pyrazole Derivatives via a Multicomponent Reaction Using Ultra Sound Irradiation
}

\author{
Bharat Baria, Denish Viradiya, Vipul Kotadiya, Rajesh Kakadiya*, \\ Anamik Shah** \\ Department of Chemistry (UGC-SAP \& DST-FIST Sponsored), Saurashtra University, \\ Rajkot - 360005, Gujarat, India \\ National Facility for Drug Discovery Center (NFDD), Saurashtra University Rajkot - 360005, India \\ ${ }^{*, \star \star E}$-mail address: kakadiyaraja@gmail.com , anamik_shah@hotmail.com
}

\begin{abstract}
Some new imidazolyl pyrazole derivatives have been synthesized using benzil, ammonium acetate, and heterocyclic aldehyde in ethanol and acetic acid under ultra sound irradiation. The obtained products using this approach are having high purity and good in yield. By conducting the reactions in ethanol, the solid products were precipitated out and could be isolated simply by filtration. The synthesized compounds were characterized by various analytical techniques such as ${ }^{1} \mathrm{H}$ and ${ }^{13} \mathrm{C}$ NMR and further supported by mass spectroscopy.
\end{abstract}

Key words: Imidazole; Ultra Sound irradiation; Imidazolyl-Pyrazole; Benzil

\section{INTRODUCTION}

Multi-component reactions (MCRs) have emerged as an efficient and powerful tool for drug discovery and modern synthetic organic chemistry due to their valued features such as atom economy (describes the conversion efficiency of a chemical process in terms of all atoms involved) and straight forward reaction design. Typically, purification of products resulting from these reactions is simple since all the organic reagents employed are consumed and incorporated into the target compound [5]. MCRs leading to interesting heterocyclic scaffolds are particularly useful for the construction of diverse chemical libraries of 'druglike' molecules. The imidazole ring is a fertile source of biologically important molecules. Compounds containing imidazole moiety have many pharmacological properties and play important roles in biochemical processes [6-12].

They are well-known as fungicides, herbicides, anti-inflammatory agents, antithrombotic agents, plant growth regulators, and therapeutic agents. Pyrazole, a fivemembered, two-nitrogen-containing heterocycles ring, is widely found as the core structure in a large variety of compounds that possess important agrochemical and pharmaceutical activities. Numerous methods have been reported for the synthesis of highly substituted imidazoles using various catalytic systems such as L-proline, $\mathrm{ZrCl}_{4}, \mathrm{InCl}_{3} \cdot 3 \mathrm{H}_{2} \mathrm{O}$, 
$\mathrm{HClO}_{4} \cdot \mathrm{SiO}_{2}, \mathrm{BF}_{3} \cdot \mathrm{SiO}_{2}, \mathrm{~K}_{5} \mathrm{CoW}_{12} \mathrm{O}_{40} \cdot 3 \mathrm{H}_{2} \mathrm{O}$, heteropolyacids, silica gel, zeolite $\mathrm{HY}$, silica gel $/ \mathrm{NaHSO}_{4}$, molecular iodine, silica sulfuric acid [13-26] etc. However, the above mentioned methods suffer from one or more disadvantages such as the use of hazardous organic solvents, expensive moisture-sensitive catalysts, tedious work-up conditions, long reaction time and large volume of catalyst loadings. So, from the above finding of biological activities associated with pyrazole and imidazole rings we have decided to combine them in one scaffold to synthesize some novel derivatives by using the simple and environmental friendly synthesis using ultra sound irradiation.

\section{RESULT AND DISCUSION}

Here, we have presented an energy efficient, rapid, and mild method for the synthesis of tri substituted imidazole derivative using acetic acid as acid catalyst under the ultra sound irradiation at room temperature. Out of range of other acid catalyst, Acetic acid has attracted much attention because of its suitable acidity, easy availability, and low cost thereby acting as a promising table top reagent.

\section{1. Reaction Scheme}

STEP - 1

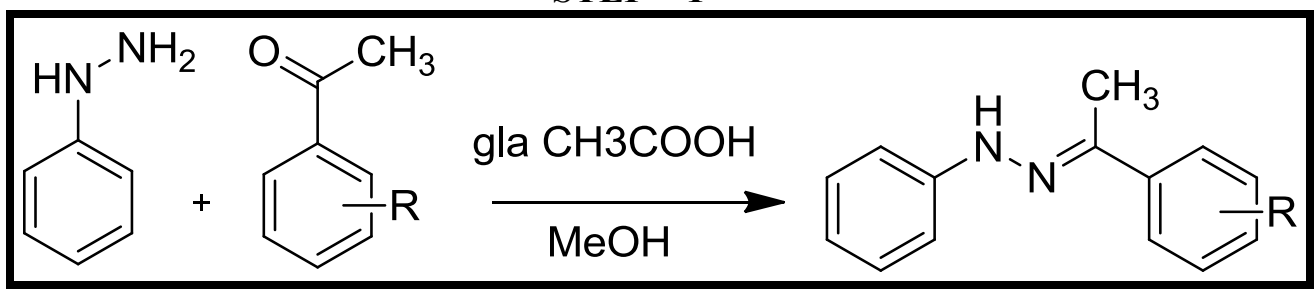

Reagents / Reaction Condition (a): Glacial acetic acid, Ethanol / Reflux, 5-6 hours. where, $\mathrm{R}=\mathrm{H}, 4-\mathrm{F}, 4-\mathrm{Cl}, 4-\mathrm{Br}, 4-\mathrm{OH}, 4-\mathrm{CH}_{3}, 4-\mathrm{NO}_{2}, 3-\mathrm{NO}_{2}$.

STEP - 2

Preparation of pyrazole aldehydes (1a-1i)

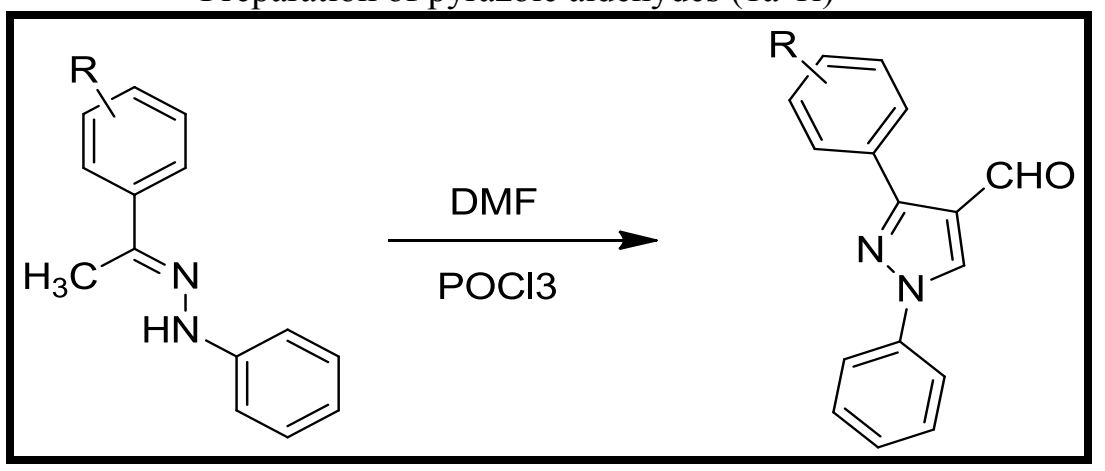

Reagents / Reaction Condition (b): DMF - $\mathrm{POCl}_{3} / 70-80{ }^{\circ} \mathrm{C}, 5-6$ hours. where, $\mathrm{R}=\mathrm{H}$, 4-F, 4-Cl, 4- $\mathrm{Br}, 4-\mathrm{OH}, 4-\mathrm{CH}_{3}, 4-\mathrm{NO}_{2}, 3-\mathrm{NO}_{2}$ 
STEP - 3

Preparation of Imidazolyl-pyrazole derivatives (3a-3i).

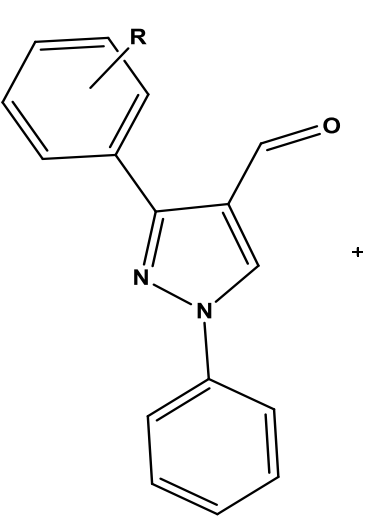

$1 \mathrm{a}$<smiles>O=C(C(=O)c1ccccc1)c1ccccc1</smiles>

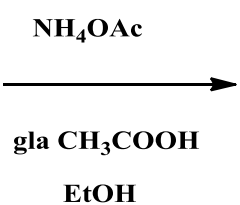

$2 \mathrm{a}$

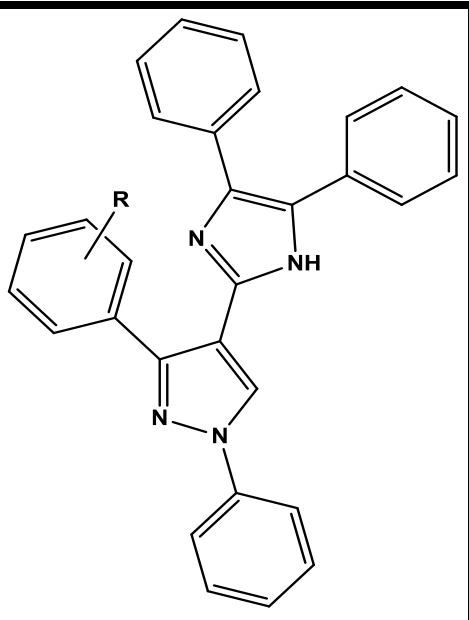

$3(\mathrm{a}-\mathrm{i})$

Table 1. Various pyrazole aldehyde used for the synthesis of Imidazolyl-pyrazole derivatives.

\begin{tabular}{|c|c|c|c|}
\hline Compound code & $\mathbf{R}$ & Time (minute) & ${ }^{\text {a }}$ Yield \% \\
\hline $3 \mathrm{a}$ & $\mathrm{C}_{6} \mathrm{H}_{5}$ & 30 & 92 \\
\hline $3 \mathrm{~b}$ & $4-\mathrm{F}-\mathrm{C}_{6} \mathrm{H}_{4}$ & 25 & 90 \\
\hline $3 \mathrm{c}$ & $4-\mathrm{Br}_{-} \mathrm{C}_{6} \mathrm{H}_{4}$ & 40 & 82 \\
\hline $3 \mathrm{~d}$ & $3-\mathrm{CH}_{3}-\mathrm{C}_{6} \mathrm{H}_{4}$ & 25 & 88 \\
\hline $3 \mathrm{e}$ & $4-\mathrm{OH}-\mathrm{C}_{6} \mathrm{H}_{4}$ & 30 & 86 \\
\hline $3 \mathrm{f}$ & $4-\mathrm{CH}-\mathrm{C}_{6} \mathrm{H}_{4}$ & 25 & 91 \\
\hline $3 \mathrm{~g}$ & $4-\mathrm{Cl}_{-} \mathrm{C}_{6} \mathrm{H}_{4}$ & 40 & 85 \\
\hline $3 \mathrm{~h}$ & $4-\mathrm{NO}_{2}-\mathrm{C}_{6} \mathrm{H}_{4}$ & 40 & 77 \\
\hline $3 \mathrm{i}$ & $3-\mathrm{NO}_{2}-\mathrm{C}_{6} \mathrm{H}_{4}$ & 35 & 80 \\
\hline
\end{tabular}

$\mathrm{a}=$ Isolated yield

\section{2. Experiment}

\section{2. Materials and methods}

Melting points were determined in open capillary tubes and are uncorrected. Mass spectra were recorded on Shimadzu GC-MS-QP-2010 model using Direct Injection Probe technique. ${ }^{1} \mathrm{H}$ NMR was determined in $\mathrm{CDCl}_{3} / \mathrm{DMSO}$ solution on a Bruker Av II $400 \mathrm{MHz}$ spectrometer. Elemental analysis of the all the synthesized compounds was carried out on Elemental Vario EL III Carlo Erba 1108 model and the results are in agreements with the 
structures assigned. Purity of the synthesized compounds was checked by merk precoated thin layer chromatography $\mathrm{G}_{254}$ and spots were visualized by iodine and UV.

\section{2. 2. Preparation of acetophenone phenyl hydrazones derivatives (Step - 1)}

Appropriately substituted acetophenones $(0.1$ mole $)$ was dissolved in $50 \mathrm{ml}$ of ethanol into $250 \mathrm{ml}$ beaker. Phenyl hydrazine $(0.1$ mole $)$ was added to above flask along with 3-4 drops of glacial acetic acid. The reaction mixture was refluxed for 3-6 hours, progress and the completion of reaction were checked by TLC using ethyl acetate : hexane $(6: 4)$ as a mobile phase. After the completion of the reaction, the reaction mixture was kept to room temperature for 1 hours and the filtered the solid crystalline product. The product was washed with ethanol and dried to give substituted acetophenone hydrazone in good yield which was used without further purification in the next step.

\section{2. 3. Preparation of Pyrazole aldehydes derivatives (1a-1i) (Step - 2)}

Dimethylformamide $(0.032$ mole $)$ was transferred into $25 \mathrm{ml}$ flat bottom flak. Phosphorous oxychloride ( 0.032 mole) was added drop wise to above flask under stirring at $0-5{ }^{\circ} \mathrm{C}$. After completion of the addition, the mixture was stirred at this temperature for $10-15$ min. freshly prepared acetophenone hydrazones $(0.015$ mole) was added to above mixture and the content was heated on oil bath for 6-9 hours.

The progress and the completion of reaction were checked by TLC using toluene: ethyl acetate (6: 4) as a mobile phase. After the reaction was completed, the reaction mixture was cooled to room temperature and poured on crushed ice. The separated solid was filtered off and it was washed with cold water. It was dried at $65^{\circ} \mathrm{C}$ and recrystallized from the mixture of DMF-Methanol to give the pure product.

\section{2. 4. General procedure for the synthesis substituted Imidazolyl-pyrazole derivatives (3a-i)}

To a stirred solution of pyrazole aldehyde (1a-1i) $(1$ mole) in ethanol: glacial $\mathrm{CH}_{3} \mathrm{COOH}(1: 1)$, benzil ( 1 mole) and ammonium acetate $(3$ mole) were added. The reaction mass was subjected to ultra sound irradiation at room temperature for 20-40 minutes. After completion of the reaction (confirmed by TLC), the obtained solid product was filtered and washed with ethanol to give pure product. The isolated compounds were characterized by ${ }^{1} \mathrm{H}$ NMR, ${ }^{13} \mathrm{C}$ NMR, elemental analysis $(\mathrm{C}, \mathrm{H}$ and $\mathrm{N}$ ) and further supported by mass spectroscopy.

\section{3. Analytical Data}

\section{4-(4,5-diphenyl-1H-imidazol-2-yl)-1,3-diphenyl-1H-pyrazole (3a)}

Characteristic: white solid, M.P.: $168-170{ }^{\circ} \mathrm{C},{ }^{1} \mathrm{H}$ NMR (400 MHz, DMSO-d6): $\delta$ 12.50 (1H,s, -NH), 8.98 (1H, s, Pyr-H), 8.05-8.10 (2H,s, Ar-H), 7.93-7.95 (2H, d, Ar-H), 7.19-7.60 $(16 \mathrm{H}, \mathrm{m}, \mathrm{Ar}-\mathrm{H}),{ }^{13} \mathrm{C}$ NMR $(100 \mathrm{MHz}, \mathrm{DMSO}-\mathrm{d} 6): \delta 148.22,146.97,144.12,142.94,140.80$ 138.91, 133.74, 131.63, 129.88, 127.81, 126.74, 124.81, 121.33, 118.31, 104.48; $\mathrm{MS}(\mathrm{m} / \mathrm{z})$ : 438 Anal. calcd for $\left(\mathrm{C}_{30} \mathrm{H}_{22} \mathrm{~N}_{4}\right) \mathrm{C}, 82.17 ; \mathrm{H}, 5.06 ; \mathrm{N}, 12.78$. Found: C, 81.18; H, 5.11; N, 12.80 . 
4-(4,5-diphenyl-1H-imidazol-2-yl)-3-(4-fluorophenyl)-1-phenyl-1H-pyrazole (3b)

Characteristic: white solid, M.P.: $182-185^{\circ} \mathrm{C},{ }^{1} \mathrm{H}$ NMR (400 MHz, DMSO-d6): $\delta 12.52(1 \mathrm{H}, \mathrm{s}$, -NH), 9.0 (1H, s, Pyr-H), 8.21 (2H,s, Ar-H), 7.93-7.95 (2H, d, Ar-H), 7.22-7.58 (16H, m, Ar$\mathrm{H}),{ }^{13} \mathrm{C}$ NMR $(100 \mathrm{MHz}, \mathrm{DMSO}-\mathrm{d} 6): \delta 163.31,160.88,148.89,139.34,139.02,136.29$, $135.08,131.00,130.41,130.33,129.66,129.29,129.03,128.63,128.14,128.00,127.61$, $127.20,126.82,126.70,126.35,118.28,114.87,114.66,112.68 ; \operatorname{MS}(\mathrm{m} / z): 456$ Anal. calcd for $\left(\mathrm{C}_{30} \mathrm{H}_{21} \mathrm{FN}_{4}\right)$ C, 78.93; H, 4.64; F, 4.16; N, 12.27 C, Found: C, 78.97; H, 4.61; N, 12.25.

\section{3-(4-bromophenyl)-4-(4,5-diphenyl-1H-imidazol-2-yl)-1-phenyl-1H-pyrazole (3c)}

Characteristic: white solid, M.P.: $175-177^{\circ} \mathrm{C},{ }^{1} \mathrm{H}$ NMR (400 MHz, DMSO-d6): $\delta 12.52(1 \mathrm{H}$, s, $-\mathrm{NH}), 9.01(1 \mathrm{H}, \mathrm{s}, \mathrm{Pyr}-\mathrm{H}), 8.14-8.16(2 \mathrm{H}, \mathrm{d}, \mathrm{Ar}-\mathrm{H}), 7.93-7.95(2 \mathrm{H}, \mathrm{d}, \mathrm{Ar}-\mathrm{H}), 7.20-7.68$ $(16 \mathrm{H}, \mathrm{m}, \mathrm{Ar}-\mathrm{H}),{ }^{13} \mathrm{C}$ NMR (100 MHz, DMSO-d6): $\delta$ 148.67, 139.29, 139.04, 136.42, 135.11, $131.83,131.04,130.96,130.30,129.74,129.54,128.70,128.23,128.09,127.71,127.33$, 126.92, 126.45, 121.61, 118.41, 112.94; $\mathrm{MS}(\mathrm{m} / \mathrm{z}): 516,\left(\mathrm{M}^{+2}\right) 518$ Anal. calcd for $\left(\mathrm{C}_{30} \mathrm{H}_{21} \mathrm{BrN}_{4}\right) \mathrm{C}, 69.64 ; \mathrm{H}, 4.09 ; \mathrm{Br}, 15.44 ; \mathrm{N}, 10.83$, Found: C, 69.67; H, 3.99; N, 10.80.

\section{4-(4,5-diphenyl-1H-imidazol-2-yl)-1-phenyl-3-(m-tolyl)-1H-pyrazole (3d)}

Characteristic: white solid, M.P.: $161-163^{\circ} \mathrm{C},{ }^{1} \mathrm{H}$ NMR (400 MHz, DMSO-d6): $\delta 12.51(1 \mathrm{H}$, s, -NH), $8.96(1 \mathrm{H}, \mathrm{s}, \mathrm{Pyr}-\mathrm{H}), 8.03-8.05(2 \mathrm{H}, \mathrm{d}, \mathrm{Ar}-\mathrm{H}), 7.92-7.94(2 \mathrm{H}, \mathrm{d}, \mathrm{Ar}-\mathrm{H}), 7.21-7.60$ $(15 \mathrm{H}, \mathrm{m}, \mathrm{Ar}-\mathrm{H}), 2.33$ (3H, s, Ar-CH 3$),{ }^{13} \mathrm{C}$ NMR (100 MHz, DMSO-d6): $\delta 149.80,139.61$, $139.21,137.55,136.41,136.38,135.58,135.25,131.12,129.77,129.72,129.48,128.71$, $128.62,128.24,128.06,128.03,127.65,127.22,126.94,126.65,126.42,118.27,112.75$; $\mathrm{MS}(\mathrm{m} / \mathrm{z})$ : 452 Anal. calcd for $\left(\mathrm{C}_{31} \mathrm{H}_{24} \mathrm{~N}_{4}\right) \mathrm{C}, 82.27 ; \mathrm{H}, 5.35 ; \mathrm{N}, 12.38$, Found: $\mathrm{C}, 82.15 ; \mathrm{H}$, $5.43 ; \mathrm{N}, 12.34$.

\section{4-(4-(4,5-diphenyl-1H-imidazol-2-yl)-1-phenyl-1H-pyrazol-3-yl)phenol (3e)}

Characteristic: creamy white solid, M.P.: $192-195{ }^{\circ} \mathrm{C},{ }^{1} \mathrm{H}$ NMR (400 MHz, DMSO-d6): $\delta$ 12.54(1Hs, -NH), 9.69 (1Hs, Ar-OH), $8.95(1 \mathrm{H}, \mathrm{s}, \mathrm{Pyr}-\mathrm{H}), 6.84-7.96(19 \mathrm{H}, \mathrm{m}, \mathrm{Ar}-\mathrm{H}),{ }^{13} \mathrm{C}$ NMR (100 MHz, DMSO-d6): $\delta$ 157.63, 150.19, 139.74, 139.20, 129.68, 129.59, 129.26, $128.47,127.46,127.24,127.01,126.46,123.42,118.15,114.79,112.15 ; \mathrm{MS}(\mathrm{m} / z): 454$ Anal. calcd for $\left(\mathrm{C}_{30} \mathrm{H}_{22} \mathrm{~N}_{4} \mathrm{O}\right) \mathrm{C}, 79.27 ; \mathrm{H}, 4.88 ; \mathrm{N}, 12.33$; O, 3.52, Found: C, 79.35; H, 4.69; N, 12.30 .

\section{4-(4,5-diphenyl-1H-imidazol-2-yl)-1-phenyl-3-(p-tolyl)-1H-pyrazole (3f)}

Characteristic: white solid, M.P.: $165-167{ }^{\circ} \mathrm{C},{ }^{1} \mathrm{H}$ NMR (400 MHz, DMSO-d6): $\delta 12.50(1 \mathrm{H}$, s, -NH), 8.97 (1H, s, Pyr-H), 8.01-8.03 (2H,d, Ar-H), 7.93-7.95 (2H, d, Ar-H), 7.20-7.59 $(15 \mathrm{H}, \mathrm{m}, \mathrm{Ar}-\mathrm{H}), 2.34$ (3H, s, Ar-CH $),{ }^{13} \mathrm{C}$ NMR (100 MHz, DMSO-d6): $\delta 149.92,139.58$, $139.16,137.54,136.38,135.23,131.09,129.76,129.71,129.46,128.70,128.60,128.22$, $128.05,128.02,127.63,127.20,126.93,126.63,126.42,118.26,112.74 ; \mathrm{MS}(\mathrm{m} / z): 452$ Anal. calcd for Anal. calcd for $\left(\mathrm{C}_{31} \mathrm{H}_{24} \mathrm{~N}_{4}\right) \mathrm{C}, 82.27 ; \mathrm{H}, 5.35 ; \mathrm{N}, 12.38$, Found: C, 82.38; H, 5.32; N, 12.39 .

\section{3-(4-chlorophenyl)-4-(4,5-diphenyl-1H-imidazol-2-yl)-1-phenyl-1H-pyrazole (3g)}

Characteristic: white solid, M.P.: 207-209 ${ }^{\circ} \mathrm{C},{ }^{1} \mathrm{H}$ NMR (400 MHz, DMSO-d6): $\delta 12.56$ (1H, s, $-\mathrm{NH}), 9.02(1 \mathrm{H}, \mathrm{s}, \mathrm{Pyr}-\mathrm{H}), 8.20-8.22(2 \mathrm{H}, \mathrm{d}, \mathrm{Ar}-\mathrm{H}), 7.93-7.95(2 \mathrm{H}, \mathrm{d}, \mathrm{Ar}-\mathrm{H}), 7.21-7.61$ $(15 \mathrm{H}, \mathrm{m}, \mathrm{Ar}-\mathrm{H}),{ }^{13} \mathrm{C}$ NMR $(100 \mathrm{MHz}, \mathrm{DMSO}-\mathrm{d} 6): \delta 148.61,139.31,139.02,136.38,135.09$, $132.92,131.44,131.02,130.01,129.75,129.53,128.72,128.25,128.07,127.72,127.32$, 126.91, 126.46, 118.38, 112.91, ; $\mathrm{MS}(\mathrm{m} / \mathrm{z}): 472,\left(\mathrm{M}^{+2}\right) 474$ Anal. calcd for $\left(\mathrm{C}_{30} \mathrm{H}_{21} \mathrm{ClN}_{4}\right) \mathrm{C}$, 76.18; H, 4.48; Cl, 7.50; N, 11.85, Found: C, 76.31; H, 4.26; N, 12.14 . 


\section{4-(4,5-diphenyl-1H-imidazol-2-yl)-3-(4-nitrophenyl)-1-phenyl-1H-pyrazole (3h)}

Characteristic: yellow solid, M.P.: 201-204 ${ }^{\circ} \mathrm{C},{ }^{1} \mathrm{H}$ NMR (400 MHz, DMSO-d6): $\delta$ 12.63 (1H, s, -NH), 9.09 (1H, s, Pyr-H), 8.51-8.54 (2H, d, Ar-H), 8.34-8.36 (2H, d, Ar-H), 7.95$7.97(2 \mathrm{H}, \mathrm{d}, \mathrm{Ar}-\mathrm{H}), 7.21-7.63(13 \mathrm{H}, \mathrm{m}, \mathrm{Ar}-\mathrm{H}),{ }^{13} \mathrm{C}$ NMR $(100 \mathrm{MHz}, \mathrm{DMSO}-\mathrm{d} 6): \delta 147.51$, $146.91,139.10,138.98,138.86,136.48,134.97,130.93,129.88,129.81,129.24,128.74$, $128.27,128.10,127.79,127.51,127.25,126.94,126.52,123.31,118.58,113.64 ; \mathrm{MS}(\mathrm{m} / \mathrm{z})$ : 483 Anal. calcd for $\left(\mathrm{C}_{30} \mathrm{H}_{21} \mathrm{~N}_{5} \mathrm{O}_{2}\right) \mathrm{C}, 74.52 ; \mathrm{H}, 4.38 ; \mathrm{N}, 14.48 ; \mathrm{O}, 6.62$, Found: $\mathrm{C}, 74.88 ; \mathrm{H}$, $4.51 ; \mathrm{N}, 14.39$.

\section{4-(4,5-diphenyl-1H-imidazol-2-yl)-3-(3-nitrophenyl)-1-phenyl-1H-pyrazole (3i)}

Characteristic: light yellow solid, M.P.: 196-198 ${ }^{\circ} \mathrm{C},{ }^{1} \mathrm{H}$ NMR (400 MHz, DMSO-d6): $\delta$ $12.64(1 \mathrm{H}, \mathrm{s},-\mathrm{NH}), 9.47$ (1H, s, Pyr-H), 9.12 (1H, s, Ar-H), 8.64-8.66 (2H, d, Ar-H), 8.28$8.30(2 \mathrm{H}, \mathrm{d}, \mathrm{Ar}-\mathrm{H}), 7.21-7.97(16 \mathrm{H}, \mathrm{m}, \mathrm{Ar}-\mathrm{H}),{ }^{13} \mathrm{C}$ NMR $(100 \mathrm{MHz}, \mathrm{DMSO}-\mathrm{d} 6): \delta 147.68$, $147.41,139.15,138.87,136.36,134.91,134.57,134.16,130.97,129.80,129.52,128.75$, $128.16,127.82,127.46,127.13,126.90,126.50,123.35,122.98,118.52,113.19 ; \mathrm{MS}(\mathrm{m} / \mathrm{z}):$ 483 Anal. calcd for $\left(\mathrm{C}_{30} \mathrm{H}_{21} \mathrm{~N}_{5} \mathrm{O}_{2}\right) \mathrm{C}, 74.52 ; \mathrm{H}, 4.38 ; \mathrm{N}, 14.48 ; \mathrm{O}, 6.62$, Found: $\mathrm{C}, 74.81 ; \mathrm{H}$, $4.54 ; \mathrm{N}, 14.28$.

\section{CONCLUSION}

We demonstrated an efficient method for the synthesis of highly substituted imidazoles using acetic acid which is inexpensive and competent acid catalyst as compare to previous reported methods. The reaction is proceed in sonicator which gives short reaction times, high yields and operationally simple are some advantages of this method. Overall, this method is very rapid with use of simple starting material and obtained high yield and purity of products without use of any further purification.

\section{ACKNOWLEDGEMENT}

We are thankful to National Facility for Drug Discovery (NFDD), jointly funded by Department of Science \& Technology, New Delhi, Government of Gujarat Industries Commissionerate \& Saurashtra University, Rajkot, Gujarat, India for providing laboratory and analytical instrumental facilities.

\section{References}

[1] P. Eilbracht, L. Barfacker, C. Buss, C. Hollmann, B.E. Kitsos-Rzychon, C. L. Kranemann, T. Rische, R. Roggenbuck, A. Schimdt, Chem. Rev. 99 (1999) 3329.

[2] L. Weber, Drug Discov. Today 7 (2002) 143.

[3] C. Hulme, V. Gore, Curr. Med. Chem. 10 (2003) 51.

[4] P.A. Tempest, Curr. Opin. Drug Discov. Devel. 8 (2005) 776.

[5] I. Ugi, Pure Appl. Chem. 73 (2001) 187.191.

[6] A. Domling, Chem. Rev. 106 (2006) 17.

[7] Heers J., Backx L. J., J. Mostmans, J. H. Van Cutsem J. Med. Chem. 22 (2003) 1979. 
[8] Hunkeler W., Mohler H., Pieri L., Polc, P. Bonetti, E. P. Cumin, R. Schaffner, R. Haefely, W. Nature 290 (1981) 514.

[9] Brimblecombe R. W., Duncan W. A. M., Durant G. J., Emmett, J. C. Ganellin, C. R., Parons M. E., J. Int. Med. Res. 3 (1975) 86.

[10] Tanigawara Y., Aoyama N., Kita T., Shirakawa K., Komada F., Kasuga M., Okumura K., Clin. Pharmacol. Ther. 66 (1999) 528.

[11] Wauquier A., Van Den Broeck W. A. E., Verheyen J. L., Janssen P. A. J., Eur. J. Pharmacol. 47 (1978) 367.

[12] S. Samai, G. C. Nandi, P. Singh, M. S. Singh, Tetrahedron 65 (2009) 1015.

[13] G. V. M. Sharma, Y. Jyothi, P. S. Lakshmi, Synth. Commun. 36 (2006) 2991.

[14] S. D. Sharma, P. Hazarika, D. Konwar, Tetrahedron Lett. 49 (2008) 2216.

[15] S. Kantevari, S. V. N. Vuppalapati, D. O. Biradar, L. Nagarapu, J. Mol. Catal. A: Chem. 266 (2007) 109.

[16] B. Sadeghi, B. B. F. Mirjalili, M. M. Hashemi, Tetrahedron Lett. 49 (2008) 2575.

[17] L. Nagarapu, S. Apuri, S. Kantevari, J. Mol. Catal. A: Chem. 266 (2007) 104.

[18] M. M. Heravi, F. Derikvand, F. F. Bamoharram, J. Mol. Catal. A: Chem. 263 (2007) 112.

[19] S. Balalaei, A. Arabanian, Green Chem. 2 (2000) 274.

[20] A. R. Karimi, Z. Alimohammadi, J. Azizian, A. A. Mohammadi, M. R. Mohmmadizadeh, Catal. Commun. 7 (2006) 728.

[21] M. Kidwai, P. Mothsra, V. Bansal, R. K. Somvanshi, A. S. Ethayathulla, S. Dey, T. P. Singh, J. Mol. Catal. A: Chem. 265 (2007) 177.

[22] A. Shaabani, A. Rahmati, J. Mol. Catal. A: Chem. 249 (2006) 246.

[23] J. Elguero, P. Goya, N. Jagerovic and A. M. S. Silva, Targets in Heterocyclic Systems Chemistry and Properties. 6 (2002) 52.

[24] Prakash Mehta, Prakash Davadra, Nirav Shah, Hitendra Joshi, International Letters of Chemistry, Physics and Astronomy 10 (2014) 74-80.

[24] Prakash Mehta, Prakash Dawedra, Vaishali Goswami, Hitendra S. Joshi, International Letters of Chemistry, Physics and Astronomy 11(1) (2014) 1-8.

[25] Prakash Mehta, Prakash Davadra, Jalpa R. Pandya, Hitendra S. Joshi, International Letters of Chemistry, Physics and Astronomy 11(2) (2014) 81-88.

[26] Dipti L. Namera, Khushal M. Kapadiya, Mitesh M. Chhatrola, Umed C. Bhoya, International Letters of Chemistry, Physics and Astronomy 11(2) (2014) 116-126. 